\title{
Gelation of aqueous gelatin solutions. II. Rheology of the sol-gel transition
}

\author{
Madeleine Djabourov, Jacques Leblond and Pierre Papon \\ Laboratoire de Physique Thermique (*), ESPCI, 10 rue Vauquelin, 75231 Paris Cedex 05, France
}

(Reçu le 25 juin 1987, accepté le 23 octobre 1987)

\begin{abstract}
Résumé. - Nous avons étudié le comportement rhéologique des solutions aqueuses de gélatine pendant le processus de gélification à plusieurs températures. Nous avons exploré la réponse dynamique du système dans le domaine viscoélastique linéaire, à plusieurs fréquences comprises entre $0.01 \mathrm{~Hz}$ et $1.5 \mathrm{~Hz}$, à l'aide du rhéogoniomètre de Weissenberg. La relation entre le module de cisaillement et le taux d'hélices montre clairement que ce dernier paramètre contrôle le processus de gélification. De l'analyse des données rhéologiques, nous déduisons une méthode pour définir le point de gel qui pourrait s'appliquer à d'autres systèmes. Finalement, nous interprétons la gélification de la gélatine dans le cadre de la théorie de percolation proposée par de Gennes et Stauffer. Les exposants pour la viscosité élastique et le module de cisaillement relaxé sont en accord avec les valeurs prévues à $3 \mathrm{~d}$.
\end{abstract}

\begin{abstract}
We have studied the rheological behaviour of aqueous gelatin solutions during the gelation process at several temperatures. The linear viscoelastic regim was investigated by dynamical measurements in the frequency range $0.01 \mathrm{~Hz}$ to $1.5 \mathrm{~Hz}$, using a Weissenberg rheogoniometer. The relation between the shear moduli and the helix amount at several temperatures puts into evidence that this structural parameter pilots the gelation process. Analysing the rheological data, we propose a method of deriving the gel point, which could be used for other systems. Finally we show that gelatin gelation can be interpreted within the framework proposed by de Gennes and Stauffer of the percolation theory. The exponents for the static viscosity and the relaxed shear modulus are in agreement with the $3 \mathrm{~d}$ predictions.
\end{abstract}

The gelation process is a mechanism which transforms a liquid solution of macromolecules or particles into an elastic well-shaped solid. One may include into this wide definition a great number of systems such as solutions of natural or synthetic polymers, solutions with reacting multifunctional components, colloidal dispersions, etc. For all the systems, the mechanism of gelation increases in a random way the number of bonds between molecules or particles, until a continuous connected network appears. The bonds can either be of a physical or a chemical origin. In the first case-hydrogen bonds, Van der Waals forces- the gels are reversible by modification of temperature, $\mathrm{pH}$, ionic force, etc... In the second case, the gels are permanently crosslinked by covalent bonds.

(*) Unité associée au Centre National de la Recherche Scientifique UA 836.
In the last decade, a great deal of theoretical work has been devoted to the analysis of the gelation process, following the ideas first proposed by de Gennes [1] and Stauffer [2]. These authors established a parallel between gelation and a percolation process. It allowed them to analyse the process of gelation by analogy with a second order phase transition, where the fraction of reacted bonds $p$ is equivalent to temperature and the gel fraction is the order parameter of the transformation. The gel point corresponds to the amount $p=p_{\mathrm{c}}$ of reacted bonds for which an infinite cluster is formed.

When approaching the gel point :

$$
\left|p-p_{\mathrm{c}}\right| \rightarrow 0
$$

Universal scaling laws have been predicted for the molecular weight of the finite clusters $\left(p<p_{\mathrm{c}}\right)$, for this mean size $\left(p<p_{\mathrm{c}}\right)$ and for the gel fraction $\left(p>p_{\mathrm{c}}\right)$. Computer simulations allow an estimation 
of the critical exponents which are then compared to the theoretical predictions.

However, the measurements of real systems are of a crucial importance for testing the validity of this theoretical approach. Most of the experiments known at the moment deal with irreversible gelation. For physical gelation, the situation is more complex. According to de Gennes [1] the physical gelation can either obey the universal laws of the "strong gelation" or be comparable to a «glass transition » which then is called «weak gelation». In order to find out an analogy between physical gelation and a percolation process, one should be able, first to define the probability of bond formation for each system. While for chemical gelation this parameter is obviously given by the fraction of reacted bonds, for physical gelation one has to identify the mechanism responsible for the aggregation of chains or particles. Then, a direct proof of the critical behaviour, for any gelling system, is given by a careful measurement of the mean cluster size $[3,4]$ or the gel fraction, as a function of $\left|p-p_{\mathrm{c}}\right|$, whenever this can be done. Besides, one can also follow the modification of the rheological properties of the medium which in fact is the most evident manifestation of the gelation phenomenon. Indeed a critical behaviour has been predicted, both for the static viscosity [1], below the gel point, and for the relaxed elastic modulus $[1,5]$ above the gel point. The measurements which are known generally deal with irreversible systems such as the copolymerisation of mono and bis-acrylamide aqueous solutions [6], the crosslinking reaction of a polydimethylesiloxane [7], the polycondensation of polyols and diisocyanate [8], and also the colloidal systems like the casein gel [9]. It appears so far that the scaling laws are able to describe these processes which are, at a microscopic level, quite different.

Very few information is available on the rheology of the sol-gel transition of physical, reversible gels. Thus, the aim of this study is to characterize the rheological behaviour of gelatin solutions during gelation.

As explained in the preceding paper, several methods can be used to prepare the gels from the solutions.

For instance, one can either progressively cool the solutions at a fixed rate or quench them at various temperatures, and follow the kinetics of the transformation. We have demonstrated that none of the methods leads to an equilibrium state of the gel. We have adopted the quenching method, which seems the more convenient way for a systematic investigation of the gelation phenomenon.

In this paper, we describe the gelation kinetics, at several quenching temperatures by rheological dynamical measurements and establish the connection with the structural modifications. We first briefly recall the rheological method and the experimental device, stressing upon the difficulties related to our sample (Sect. 1). Section 2 deals with the experimental results : in 2.1, we show the influence of temperature on the shear moduli of the gels, measured at one frequency, in 2.2 we display the frequency dependence of the shear moduli, for one temperature of gelation. In section 3 , we examine the data within the framework of the percolation theory. We conclude in section 4 by discussing the method of analysis and proposing a definition of the gel point, from rheological measurements, which could be adopted for other gelling systems.

\section{Experimental procedure.}

The rheological measurements have been done with a Weissenberg Rheogoniometer R17 (SANGAMO), in oscillatory shear. We used a cone and plane geometry (radius $2.5 \mathrm{~cm}$ ). The plane has been modified in order to realize a precise thermal regulation of the samples and to avoid evaporation [10]. Temperature was regulated within $\pm 0.05^{\circ} \mathrm{C}$ and kept constant for periods of 7 to 8 hours.

The method consists of imposing oscillations (of variable amplitudes and frequencies) to the lower plate and to measure the displacement of the upper plate. Under standard conditions, one may assume that the angular movement of the lower plate represents the strain $\varepsilon$ while the movement of the upper plate is proportional to the stress $\sigma$ (the amplitude depending on the dimensions of the plate and on the characteristic stiffness of the torsion bar).

In the linear viscoelastic regime (small deformations), the stress is proportional to the strain, and determines the complex shear modulus by :

$$
G^{*}=\sigma / \varepsilon=G^{\prime}+i G^{\prime \prime}=|G|(\sin \varphi+i \cos \varphi) .
$$

$G^{\prime}$ is the storage modulus, $G^{\prime \prime}$ the loss modulus, $\varphi$ is the loss angle. For a Newtonian liquid, $G^{\prime}=0$ and the displacements of the two plates are out of phase $(\varphi=\pi / 2)$. For a Hookean solid, $G^{\prime \prime}=0$, and the plates move in phase $(\varphi=0)$.

Generally, a polymer solution or a melt has a complex shear modulus, both components $G^{\prime}$ and $G^{\prime \prime}$ depending on frequency. The rheological characterization of the medium must be done on a wide range of frequencies.

When studying a gelation process by rheological measurements, several kinds of difficulties are encountered :

a) In most cases, gelation is a kinetic process. There is no means of stopping the reaction and analysing the data step by step, in successive equilibrium states. Thus usually one tries to slow down the process. For instance, in the case of gelatin gels, by raising the temperature by only a few degrees (above 
$25^{\circ} \mathrm{C}$ ), one can considerably change the kinetics up to a factor of 10). So, we have been able to analyse the kinetics at several frequencies $f$ between 0.01 and $1.5 \mathrm{~Hz}$. The lower limit is related to the kinetics of the transformation ( 1 period $=100 \mathrm{~s})$ and also to the smallness of the moduli.

b) The measurements have to be done in the linear regime (small deformations). One has to satisfy the contradictory requirements to keep the deformation as small as possible and to be able to measure the torque, applied to the sample (the stress). This aspect represents the greatest difficulty that we had to overcome, for gelatin gelation. An additional complication came from the nature of the bonds which are reversible. The junctions can easily be destroyed by the shear deformation. However, there is not any obvious indication when this happens to be the case. In order to find the right conditions, we have done comparative experiments, at different shear strains and frequencies. When the measurements disturbed gelation, we noticed that the increase the shear modulus, versus time, was significantly delayed. At equal amplitudes of the shear strains, the lowest frequencies $(f \approx 0.01 \mathrm{~Hz})$ are the most perturbative, possibly because the duration of a period of oscillation then is the longest. In most cases, the shear stress (defined by the ratio between the angular amplitude of the strain to the cone angle) was kept around $10 \%$. At the higher frequencies $(0.15$ and $1 \mathrm{~Hz})$ the sample supports higher stresses $(40 \%)$, especially at the beginning of gelation. For the smallest moduli $(0.1$ to $1 \mathrm{dyn} / \mathrm{cm}^{2}$ ), every time we could, we have accumulated several oscillations, in order to average the signals. We used a NICOLET 4094 numerical oscilloscope and stored the data on floppy disks.

The relative amplitudes and phase lag between the sine waves were analysed by standard programs. The accuracy for the former is of the order of a few percent and for the latter better than $0.02 \mathrm{rad}$.

Last, we recall that the sample preparation was described in the preceding paper. The concentration used for the rheological measurements is $4.7 \% \mathrm{~g} / \mathrm{g}$. The solvent is water $0.1 \mathrm{M} \mathrm{NaCl}$, with a small amount of antibacterial $\mathrm{NaN}_{3}$. The $\mathrm{pH}$ was adjusted to 7 .

\section{Rheological measurements.}

2.1 GELATION AT DIFFERENT TEMPERATURES. The influence of temperature on the kinetics of gelation is quite strong. Thus the temperature range where one can follow the rheological modifications is limited to a few degrees. We report first the increase of the shear modulus $|G|$ versus time for periods ranging between 30 and $400 \mathrm{~min}$, at several temperatures (between 24 and $28{ }^{\circ} \mathrm{C}$ ) and at one frequency $f=0.15 \mathrm{~Hz}$. In figure $1 \mathrm{a}$, one can see that the rate of increase of the shear modulus is divided almost by ten, when temperature is raised by only $4{ }^{\circ} \mathrm{C}$. The phase analysis shows that the loss modulus $G^{\prime \prime}$ which can be measured at the beginning of the process is very low (a few dyn $/ \mathrm{cm}^{2}$ ). We found [10] that this component first increases to a slight maximum $\left(G^{\prime \prime} \approx 8 \mathrm{dyn} / \mathrm{cm}^{2}\right)$, then decreases or remains constant. Eventually, the loss component represents only a few percent of the storage modulus of mature gels. Thus, at the scale chosen in figure 1a, the moduli are almost entirely elastic and independent on the frequency.

For the same thermal treatments, we have recorded the modifications on the optical rotation of the samples as described in the precedent paper, and calculated the corresponding helix amounts. These measurements are given in figure $1 \mathrm{~b}$, versus time : the highest helix amount reported is $\chi=14 \%$ for the gel quenched at $T=24{ }^{\circ} \mathrm{C}$. It corresponds to a shear modulus of the order of $1500 \mathrm{dyn} / \mathrm{cm}^{2}$. Finally in figure 1c, we have plotted the shear modulus $|G|$ as a function of the helix amount, for all the temperatures investigated. One finds out that the different kinetics condense into an almost unique $G(\chi)$ plot. The small shifts which appear between the different sets of measurements could be due to unavoidable discrepancies in temperature regulation $\left(<0.1^{\circ} \mathrm{C}\right)$ between the two kinds of experiments. No systematic shift is observed. When the shear moduli are smaller than $10 \mathrm{dyn} / \mathrm{cm}^{2}$, the data is not reported. It will be analysed in the next section.

The helix amount appears as the main structural parameter which determines the rheological properties of the medium at the beginning of gelation. The relation between the shear modulus and the helix amount is not a linear one :

a) for helix amounts below $\chi \approx 7 \%$ the medium is predominantly viscous and has a low modulus;

b) for helix amounts above $\chi \approx 7 \%$, the modulus increases strongly with the helix amount and the medium is almost purely elastic ;

c) the viscoelastic range situated around $\chi \approx 7 \% \pm 1 \%$ will be analysed in the next section.

The uniqueness of the curve relating $|G|$ to $\chi$ proves that the structures nucleated at the different temperatures are almost identical, in the helix range $\chi<15 \%$. Temperature appears as a factor modifying only the kinetics of the transformation, as it does for a chemical reaction. However, contrary to a chemical reaction, the rate of the coil $\rightarrow$ helix conversion increases when the temperature is lowered. Also, the local structure of a helical region, as stated in the preceding paper, is obviously much more complicate than a covalent bond. The rheological measurements prove that the helical sequencies play the role of junctions or that the probability for a chain to be bound to another one is directly related to the probability of forming helices. 

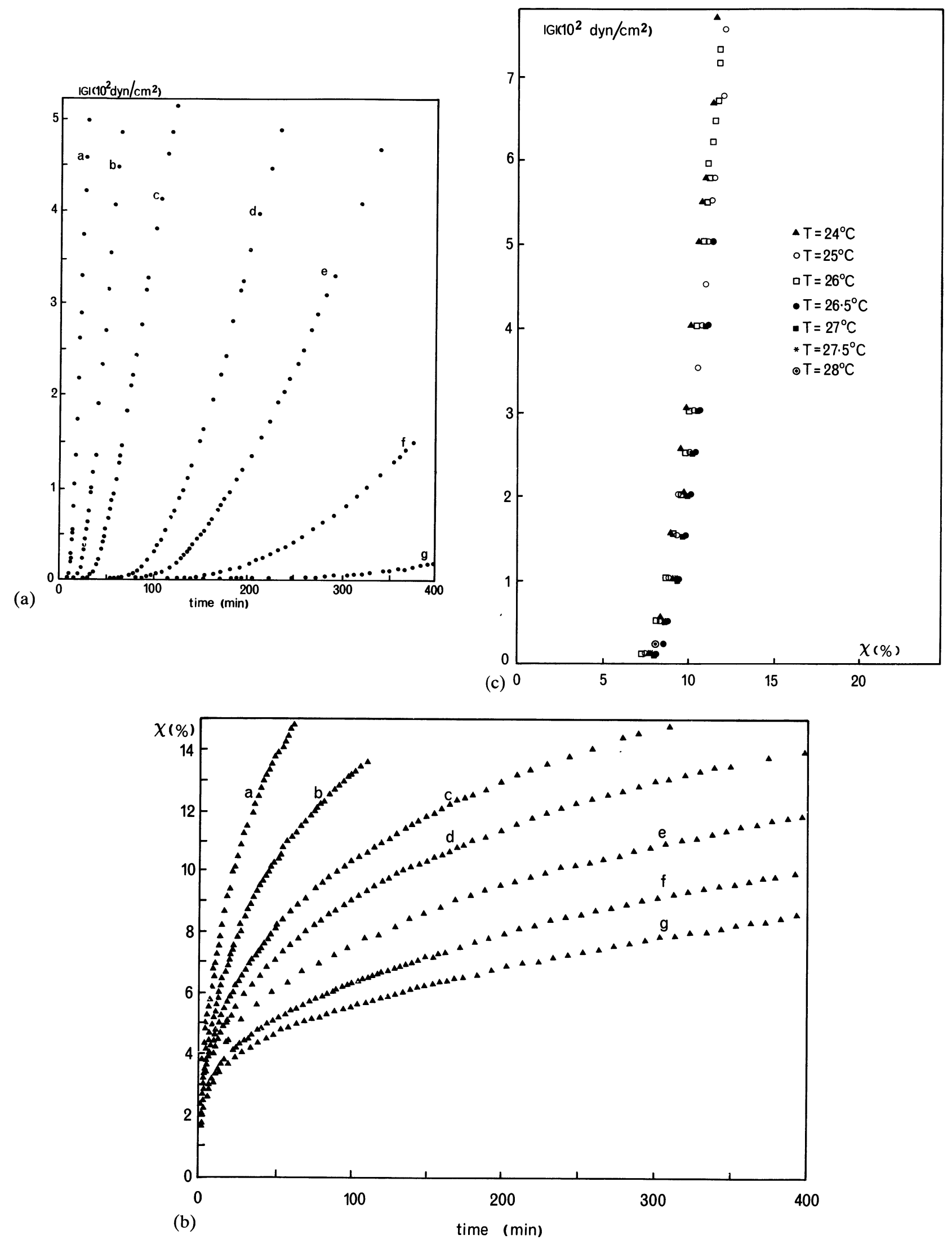

Fig. 1. - Increase of the shear modulus during gelation at different temperatures. 1a) time dependence of the shear modulus $|G|$, at $f=0.15 \mathrm{~Hz}$, for different temperatures : a) $T=24^{\circ} \mathrm{C} ;$ b) $T=25^{\circ} \mathrm{C}$; c) $T=26^{\circ} \mathrm{C}$; d) $T=26.5^{\circ} \mathrm{C}$; e) $T=27^{\circ} \mathrm{C}$; f) $T=27.5^{\circ} \mathrm{C}$; g) $T=28^{\circ} \mathrm{C}$. 1b) time dependence of the helix amount for the same temperatures. 1c) relation between the shear modulus $|G|$ and the helix amount $\chi$ (all the measurements for $c=4,7 \% \mathrm{~g} / \mathrm{g}$ ). 
So, one may consider that the structural parameter $x$ for the gelatin gels is the equivalent of the fraction of reacted bonds $\mathrm{p}$ for a chemical reaction (at least, when $\chi<15 \%$ ).

\subsection{FREQUENCY DEPENDENCE OF THE SHEAR MOD-}

ULI. - We examine now the viscoelastic behaviour which is present at the beginning of gelation.

In order to analyse it, one needs to measure the loss modulus $G^{\prime \prime}$ and the storage modulus $G^{\prime}$ on a wide range of frequencies, at successive time intervals. However, as gelation affects the flowing properties of the solutions, the modifications of the rheological parameters are expected to occur mostly in the low frequency range. The investigation at low frequencies is the most important one.

We have chosen the quenching temperature $T=27.4^{\circ} \mathrm{C}$ (or $T=27.5^{\circ} \mathrm{C}$ ). The kinetics being slow enough we measured the moduli at different frequencies : $0.015<f<1.5 \mathrm{~Hz}$. We report in figure 2 the results obtained in four independent runs, during $200 \mathrm{~min}$. At the beginning, only the loss modulus $G^{\prime \prime}$ is measurable. The sine waves are out of phase. $G^{\prime \prime}$ increases with time. Then, the storage modulus $G^{\prime}$ becomes measurable $(\varphi \neq \pi / 2)$, starting with the higher frequency $(f=1.58 \mathrm{~Hz})$, at $t=70 \mathrm{~min}$.

Then, both $G^{\prime}$ and $G^{\prime \prime}$ increase with time, while $G^{\prime}$ is smaller than $G^{\prime \prime}$, for a certain period. However, $G^{\prime}$ increases more rapidly than $G^{\prime \prime}$ and after passes beyond it. The crossing points are showed by circles, in figure 2. The corresponding times are an increasing function of the frequency as it is summarized in table I.

Table I. - Times corresponding to $G^{\prime}=G^{\prime \prime}$ for different frequencies, during gelation at $T=27.4^{\circ} \mathrm{C}$.

\begin{tabular}{ccccc}
\hline$f(\mathrm{~Hz})$ & 0.015 & 0.05 & 0.5 & 1.58 \\
\hline $\begin{array}{c}\text { time } \\
(\mathrm{min})\end{array}$ & 123 & 127 & 142 & 160 \\
\hline
\end{tabular}

Then, $G^{\prime}$ still increases and the loss angle $\varphi$ becomes smaller $(\varphi \approx 0.02 \mathrm{rad})$. After 170 or $180 \mathrm{~min}$, we are no more able to measure very accurately the loss modulus $G^{\prime \prime}$ as it appears as the product of a large amplitude factor $|G|$ by the sin term, which tends to zero. This moment seems to correspond to a maximum [10] of $G^{\prime \prime}$ or to its levelling off. As we already pointed out, for mature gels $G^{\prime \prime}$ represents only a few percent of the total shear modulus.

We are now in position to reconstitute the frequency spectrum at different instants of gelation. The loss modulus $G^{\prime \prime}$ is given in figure 3 in a double-

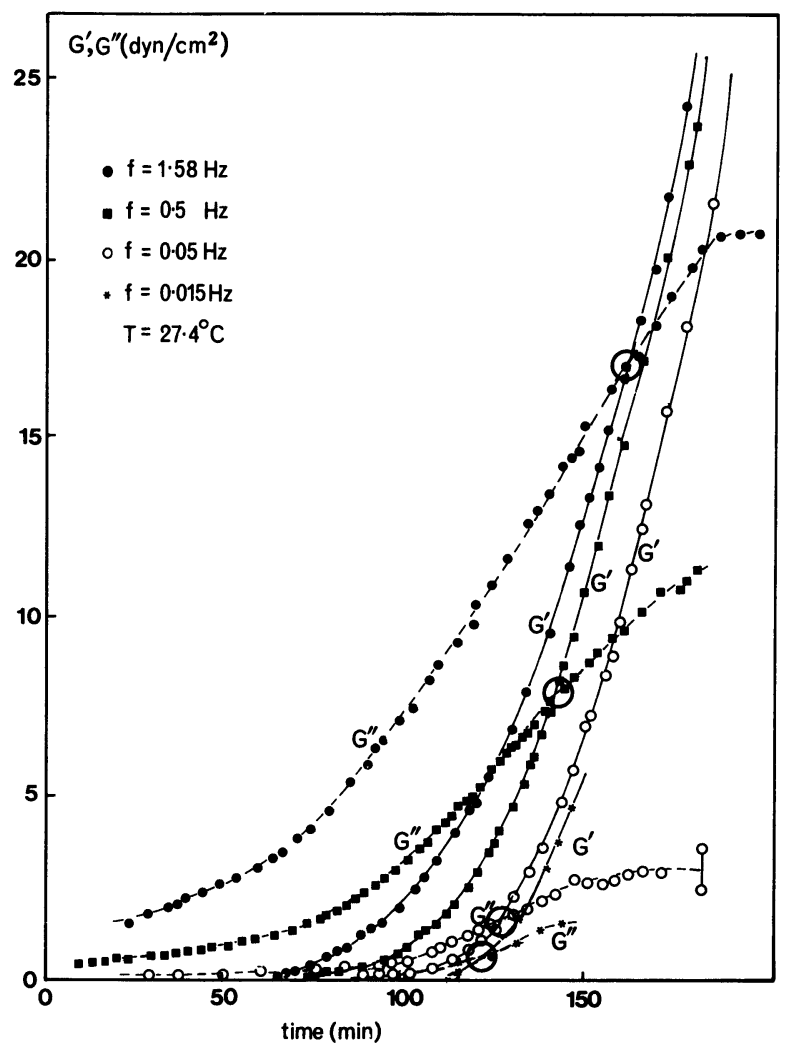

Fig. 2. - Time dependence of the loss modulus $G^{\prime \prime}$ and of the storage modulus $G^{\prime}$ for different frequencies. The quenching temperature is $T=27.4^{\circ} \mathrm{C}$. Lines are drawn to guide the eye : continuous lines for $G^{\prime}$, dashed lines for $G^{\prime \prime}$.

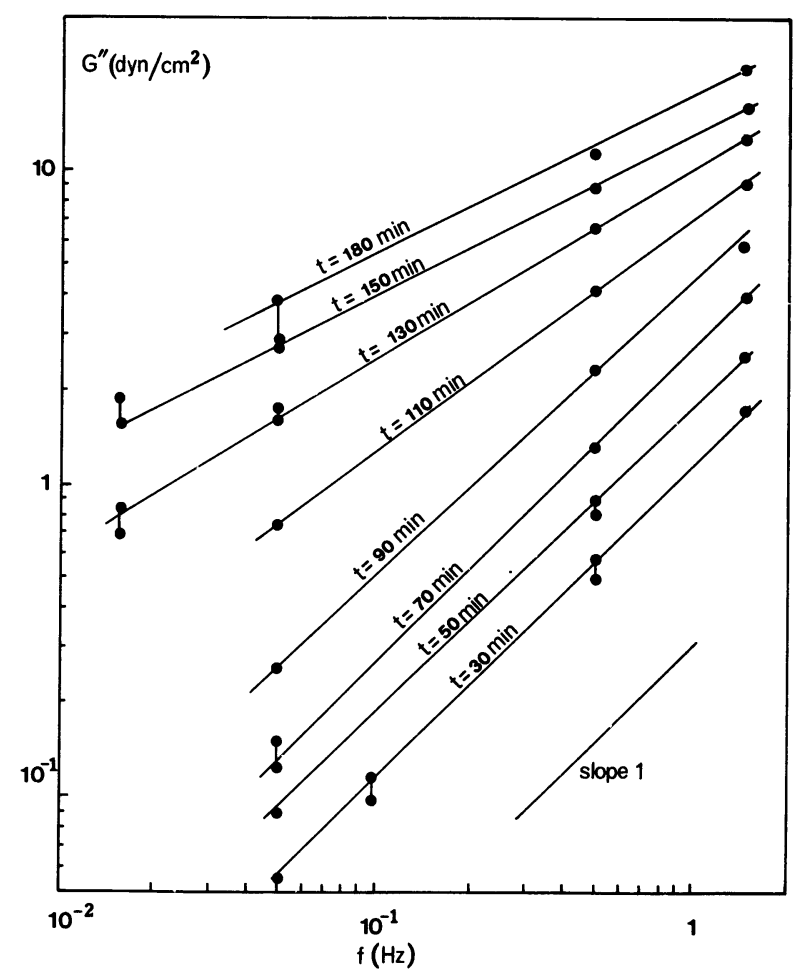

Fig. 3. - Frequency dependence of the loss modulus $G^{\prime \prime}$ at different instants of gelation, at $T=27.4^{\circ} \mathrm{C}$. 
logarithmic plot, over two decades of frequencies. Up to $90 \mathrm{~min}, G^{\prime \prime}$ is proportional to the frequency. The ratio :

$$
G^{\prime \prime} / \omega=\eta, \quad \omega=2 \pi f
$$

determines the static viscosity $\eta$ of the viscoelastic solution, indicating that at this low frequency limit, the macromolecular solution behaves as a Newtonian liquid. This implies that the characteristic relaxation times $\tau$ of the system are much smaller than the inverse of $\omega: \tau<0.1 \mathrm{~s}$.

As gelation progresses, the slope of $\log G^{\prime \prime}$ versus $\log f$ decreases, as the relaxation spectrum moves towards longer times : $\tau \approx 1 / 2 \pi f$. The static viscosity should in principle, be measurable at much lower frequencies as long as the medium is a viscoelastic liquid. However in a kinetic experiment, one is limited by the rate of the transformation.

Examining $G^{\prime}$ during the same period, we first notice in figure 4 that the moduli at the beginning of gelation vary nearly as the square of the frequency :

$$
G^{\prime} \approx f^{2}
$$

implying here again that we explore the low frequency limit of a viscoelastic liquid (Maxwell model).

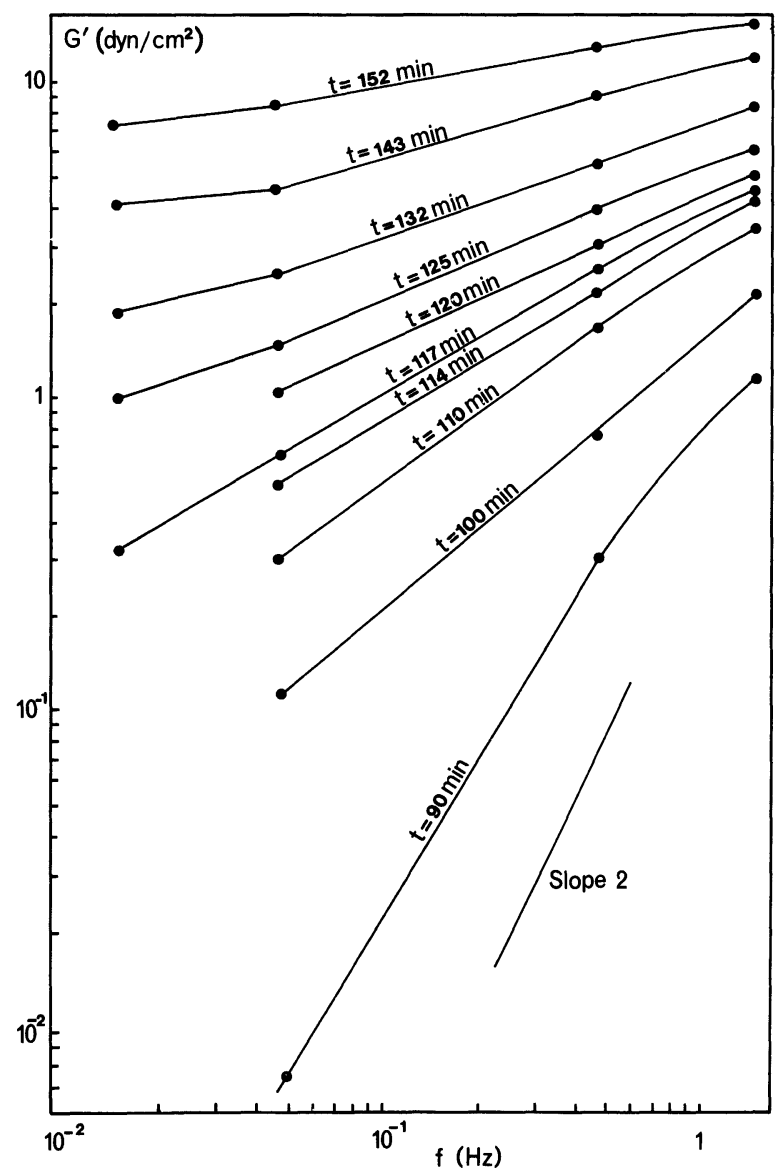

Fig. 4. - Frequency dependence of the storage modulus $G^{\prime}$ at different instants of gelation at $T=27.4^{\circ} \mathrm{C}$.
As gelation proceeds, the slope decreases, but for a certain period (up to $120 \mathrm{~min}$ ), the storage modulus still extrapolates to zero, at zero frequency. After $120 \mathrm{~min}$, a modification appears in the frequency range $0.01<f<0.05 \mathrm{~Hz}$ which indicates a non zero relaxed modulus. The medium has turned to a viscoelastic solid. To better analyse this effect, we have repeated these experiments with a systematic investigation of the low frequency limit of the storage modulus. The results are given in figure 5 for one experiment, during which the different frequencies were used, alternatively. The temperature was slightly higher $T \approx 27.5^{\circ} \mathrm{C}$. The presence of the relaxed component is clearly identified in this particular experiment, after $140 \mathrm{~min}$, by the flattering of the curves in the lowest frequency ranges. As the same moment, the storage modulus becomes larger than the loss modulus, for the lowest frequency $(f \approx 0.015 \mathrm{~Hz})$. Referring to a simple mechanical model of a viscoelastic solid, one may consider the

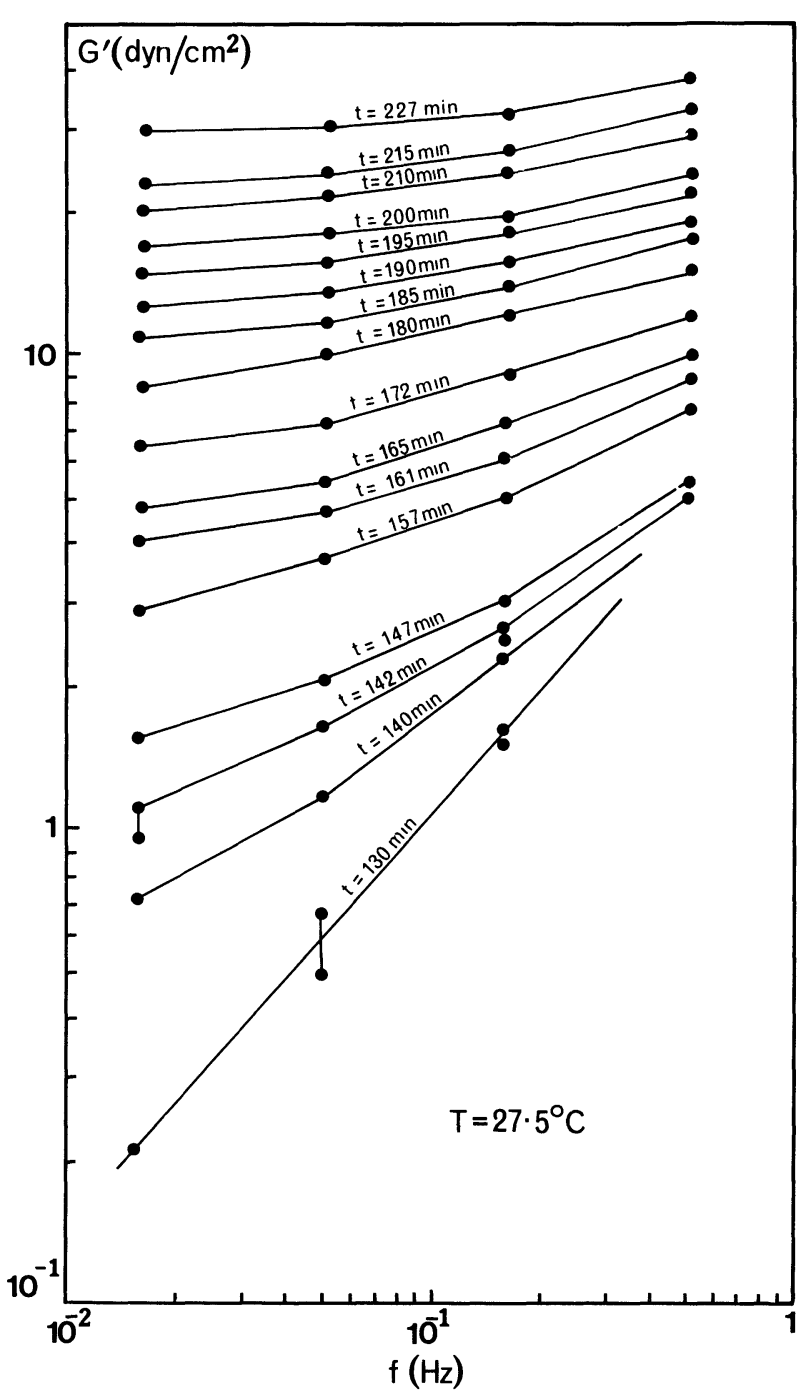

Fig. 5. - Low frequency investigation of the storage modulus $G^{\prime}$, at different instants of gelation, at $T=$ $27.5^{\circ} \mathrm{C}$. 
Zener model which is built of a spring and a dash pot, in parallel with an other spring $G_{\mathrm{r}}$. The dynamic response of such a model [11] shows that in the low frequency limit of the viscoelastic range $G^{\prime}$ becomes equal to $G^{\prime \prime}$, indicating the beginning of a rubber like plateau extended below this limit. There, $G^{\prime}$ is frequency independent, equal to $G_{\mathrm{r}}$ and it is larger than $G^{\prime \prime}$ (see Fig. 6). Although the relaxation spectrum of a gelling solution is much more complex than the response of a Zener model, we observe the same trend in the low frequency limit.

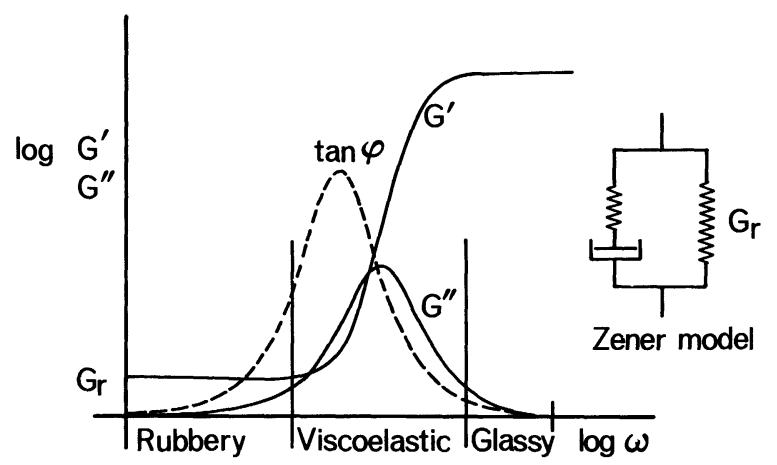

Fig. 6. - Frequency dependence of the shear moduli, $G^{\prime}$ and $G^{\prime \prime}$, for a viscoelastic solid (the Zener model).

Thus, we propose the following criteria to fix the gel time $t_{\mathrm{g}}$ :

a) examination of the frequency dependence of the storage moduli $G^{\prime}$ in the lowest frequency range which is compatible with the kinetics;

b) comparison between $G^{\prime}$ and $G^{\prime \prime}$;

c) the gel time $t_{\mathrm{g}}$ is determined by the moment when $G^{\prime}$ becomes equal to $G^{\prime \prime}$ at the lowest frequency ; it corresponds as well to the moment when a plateau begins to show in the $G^{\prime}(f)$ plot.

Because of the limited range of frequencies which can be used during a kinetic process and the limited number of measurements, we have allowed an error bar on the gel time which we consider to be of the order of $\pm 5 \mathrm{~min}$ in our experiments.

Summarizing, we have taken :

$$
t_{\mathrm{g}}=120 \pm 5 \mathrm{~min}
$$

for the set of data given in figures 2,3 and 4 ;

$$
t_{\mathrm{g}}=140 \pm 5 \mathrm{~min}
$$

for the data in figure 5 .

As we have been able to relate the rate of gelation to the coil $\rightarrow$ helix conversion, we shall now analyse the rheological parameters in terms of the helix content of the solutions.

\section{Theoretical analysis.}

3.1 The PERCOlation MODEL. - It appears from the preceding sections that the gelation phenomenon can be interpreted as a transition well-defined, in time, from a liquid like to a solid like behaviour. A careful examination of the frequency dependence of the viscoelastic moduli is necessary in determining the gel point, from rheological arguments. The relation between the shear modulus and the helix amount puts also clearly into evidence the threshold which separates these two domains.

These observations led us to further analyse the rheological data using the theoretical framework proposed by de Gennes and Stauffer $[1,2]$, that is to say the percolation model.

The percolation theory deals with the effects of increasing interconnections in random media. Apart gelation, it has been applied to a great number of physical problems [12], among which the flowing of liquids in porous media, the transition conductor insulator in composite materials, and also the spread of a disease to an epicemic...

The percolation transition is equivalent to a second order phase transition where the fraction of connected bonds corresponds to temperature : for instance the vapor-liquid critical point or the Curie-point of the paramagnetic to ferromagnetic phase transition [13] are the analogous of the gel point.

In a gelation process, the growth of clusters of increasing sizes, and finally the appearance of the infinite cluster lead to important modifications of the rheological properties of the medium.

The predictions are that, below the gel point, the static viscosity diverges, and, above the gel point, the relaxed modulus increases with a power law.

The divergence of static viscosity as the gel point, $p=p_{\mathrm{c}}$ is approached from below, can be expressed as :

$$
\eta \approx\left(1-p / p_{\mathrm{c}}\right)^{-k}, \quad p<p_{\mathrm{c}}
$$

Several models have been proposed to evaluate the value of $k$ [13]. Using a Rouse approximation, de Gennes [14] calculated the viscosity of a gelling solution, where the solvent flows without rotation of the clusters. In this case, $k=1.3$. Besides, the analogy with a network with supraconducting and dielectric junctions leads to $k=0.7$, in the three dimensional space. The classical or mean field theory gives a finite value for the viscosity, at the gel point.

The theoretical predictions for the increase of the relaxed modulus $\mathrm{E}$ from zero, above the gel point, are summarized by the equation :

$$
E \approx\left(p / p_{\mathrm{c}}-1\right)^{t}, \quad p>p_{\mathrm{c}} .
$$

By analogy to the conductance of a random resistor network, the exponent $t$ is estimated $[15,18]$ to be $t=1.8$ to 2.0 , in agreement with the numerical simulations. The classical or mean field theory which describes the gelation process as the growth of a tree-like cluster [16] predicts [17] $t=3$. Recently, 
Kantor and Webman [5] proposed a « vector percolation » theory for a lattice of springs which possess angular forces between bonds. This mechanical analogy gives an exponent for elasticity which would vary between $t=3.5$ and 4 .

The choice of the percolation theory for analysing a physical gelation process is non trivial. This theory is meant to describe a succession of equilibrium or static states, the kinetic aspects being not taken into account. Recently, computer simulations of the kinetics of irreversible 3-dimensional gelation (freeradical copolimerization) have been performed [19]. It appears that the critical exponents for the average molecular weight or the gel fraction versus the conversion factor $\left|p-p_{\mathrm{c}}\right|$ are identical with the random percolation values. The presence of non diffusive solvent molecules does not affect this behaviour [20]. Nevertheless, the simulations indicate that the kinetic (irreversible) gelation belongs to a different universality class, than random percolation (the amplitude ratios are different [19]). Besides, different kinetic assumptions (for instance, the coagulation model [21]) are likely to modify the critical behaviour. However, the latter do not seem to be relevant for the gelation process by free radical copolymerization [19]).

Thus, the observations on real systems are needed in order to prove the validity of certain hypothesis and to give support to the overall analysis.

3.2 INTERPRETATION OF THE RESULTS. - We have interpreted our results on gelatin gelation in as a critical phenomenon, and evaluated the exponents $k$ and $t$ [Eqs. (4) and (5)] from the experimental data. The definition of the gel point being given in the precedent section, we have calculated the equivalent helix content, using the data given in figure $1 \mathrm{~b}$. In both sets of rheological experiments (Sect. 2.2) the temperature was of the order of $T=27.4^{\circ} \mathrm{C}$ or $T=27.5^{\circ} \mathrm{C}$. As it is not possible to achieve an absolute identity of temperatures between the rheological and the polarimetric measurements, we have taken the data corresponding to the $\chi(t)$ curve, for $T=27.5^{\circ} \mathrm{C}$. So, for the experiments reported in figures 2,3 and 4 , the gel time $t_{\mathrm{g}}=120 \pm 5 \mathrm{~min}$, gives a critical helix content, at the gel point, $\chi_{\mathrm{c}}$ :

$$
\chi_{\mathrm{c}}=6.6 \% \text { or } \chi_{\mathrm{c}}=6.7 \% \text { or } \chi_{\mathrm{c}}=6.78 \%
$$

while for the data given in figure 5, the gel time is $t_{\mathrm{g}}=140 \pm 5 \mathrm{~min}$ and the critical helix content is :

$$
\chi_{\mathrm{c}}=6.95 \% \text { or } \chi_{\mathrm{c}}=7 \% \text { or } \chi_{\mathrm{c}}=7.1 \% \text {. }
$$

One notices that the differences in helix content for the time interval allowed as incertitude bar, is rather low.

We are now able, first, to plot the ratio $G^{\prime \prime} / \omega$ as a function of the distance to the gel point, given by $\left(1-\chi / \chi_{\mathrm{c}}\right)$ using the data between 40 and $100 \mathrm{~min}$, at $f=0.5 \mathrm{~Hz}$ (see Fig. 2). The plot is given in figure 7 in a double logarithmic scale. The three different critical helix contents are reported. The following observations can be drawn :

a) From the measurements corresponding to the first $90 \mathrm{~min}$ of gelation, one can derive a critical exponent $k=1.48 \pm 0.08$, when the distance to the gel point is :

$$
0.1 \leqslant \Delta \chi / \chi_{\mathrm{c}} \leqslant 0.3
$$

In figure 3 , these experimental points are shown to belong to the Newtonian regime.

b) More closer to the gel point, when $\Delta \chi / \chi_{\mathrm{c}} \leqslant 0.1$, a change of slope appears, which actually coincides with the non Newtonian behaviour of the viscosity.

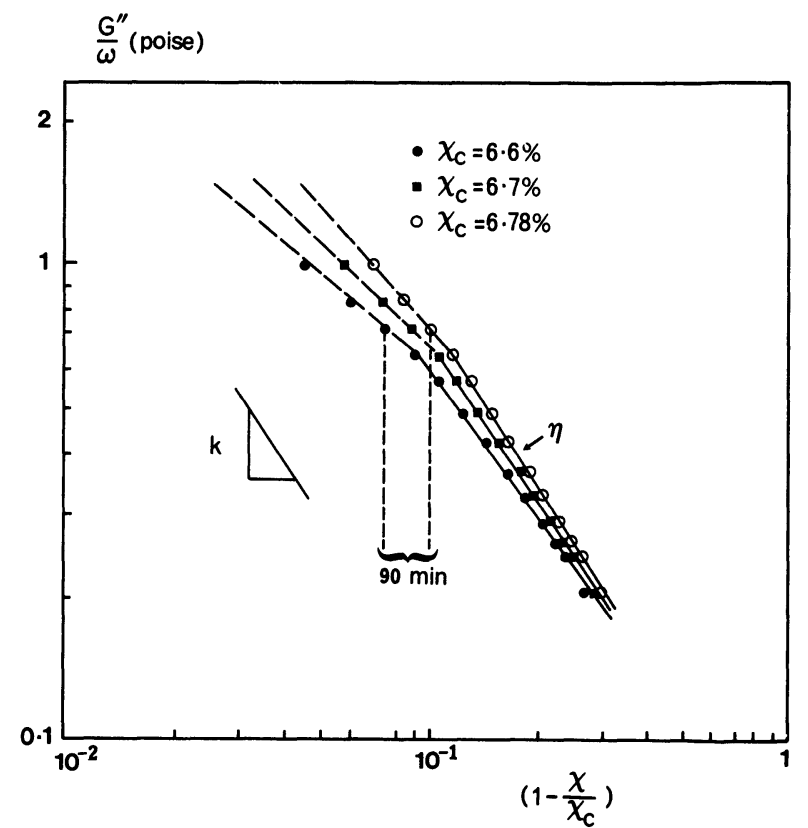

Fig. 7. - Critical behaviour of the Newtonian viscosity $\eta$. The exponent $k$ is equal to $1.48 \pm 0.08$.

We may conclude, here, that the critical behaviour is consistent with our observations, as far as we are able to measure a viscosity independent on frequency. The values of $k$ that we determined are in agreement with de Gennes predictions (14).

Finally, we analyse the evolution of the storage moduli $G^{\prime}$ above the gel point, in order to derive the exponent $t$ (Eq. (5)). We use the data from figure 5 at $f=0.015 \mathrm{~Hz}\left(T=27.5^{\circ} \mathrm{C}\right) . G^{\prime}$ is plotted in figure 8 in a double logarithmic scale as a function of the distance $\left(\chi / \chi_{\mathrm{c}}-1\right)$ to the gel point. The three values of the threshold $\chi_{\mathrm{c}}$ are shown.

One may note the following points :

a) The exponent $t$ can be estimated with a fairly good precision from the measurements of $G^{\prime}$ betwen 


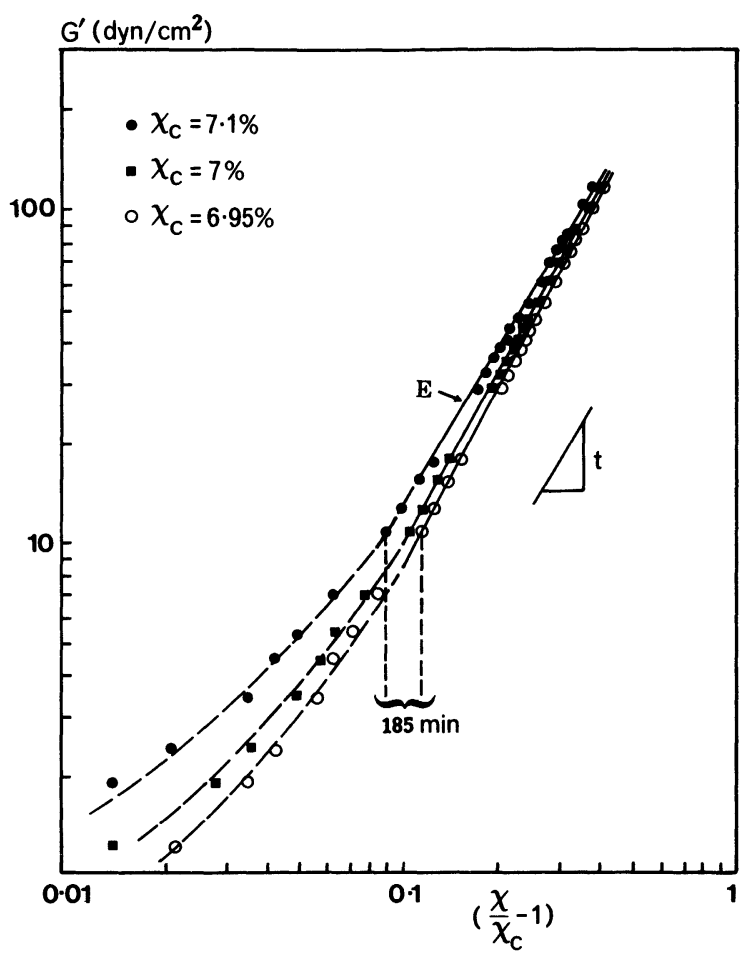

Fig. 8. - Critical behaviour of the relaxed shear modulus $E$. The exponent $t$ is equal to $1.82 \pm 0.15$.

185 and $350 \mathrm{~min}$, corresponding to an interval $0.1 \leqslant \Delta \chi / \chi_{\mathrm{c}} \leqslant 0.5$. During this period $G^{\prime}$ increases over a decade (10 to $\left.100 \mathrm{dyn} / \mathrm{cm}^{2}\right)$. Turning back to figure 5, we notice that during this period $G^{\prime}$ is almost independent on frequency, especially in this lower frequency range. Thus the values measured at $f=0.015 \mathrm{~Hz}$ can be taken as the relaxed storage modulus.

The exponent $t$ is estimated to :

$$
t=1.82 \pm 0.15
$$

in agreement with the value predicted by de Gennes [15] by analogy with the conductance of a random network.

b) Closer to the gel point, $0.01<\Delta \chi / \chi_{\mathrm{c}}<0.1$, the relaxation effects lead again to a departure from the theoretical predictions. The values measured for $G^{\prime}$ are higher than the relaxed moduli, which is obviously seen in figure 5 also.

\section{Discussion and conclusions.}

The rheological measurements during the gelation process of gelatin are consistent with the definition of a gel point. This one can be practically located by closely examining the frequency dependence of both the loss modulus and the storage modulus. The procedure that we propose is summarized in figure 9 and can be generalized to other gelling systems. It requires that close to the gel point :

$$
G^{\prime}=G^{\prime \prime} \text {. }
$$

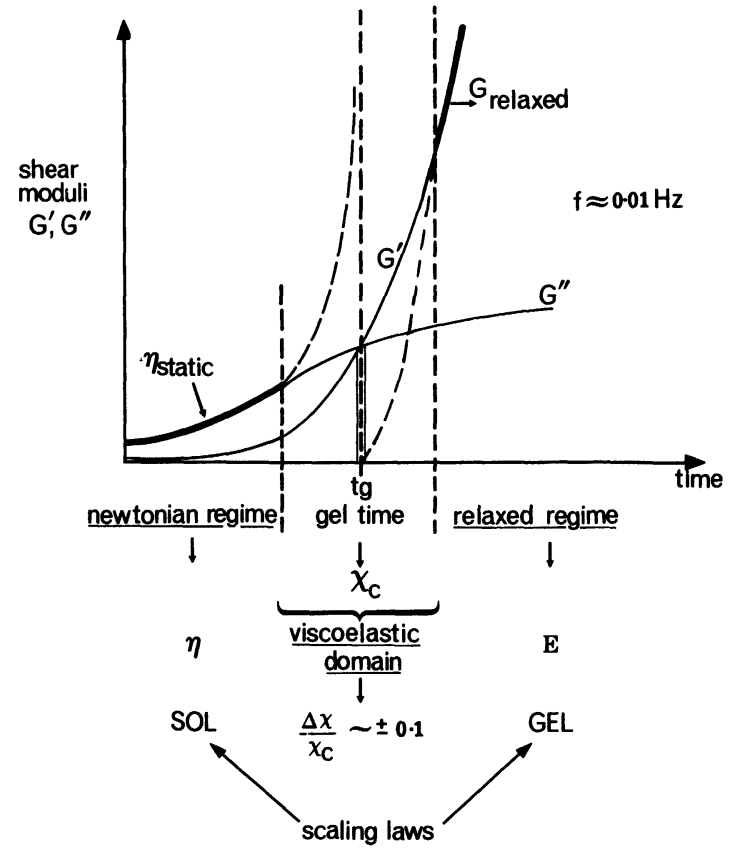

Fig. 9. - Determination of the gel point and of the critical exponents from rheological measurements.

While this criterion has already been proposed in the literature [7], the condition was believed to be totally frequency independent. Our experimental data shows clearly that this is not the case for all gelling systems. For gelatin, the crossing of $G^{\prime}$ and $G^{\prime \prime}$ obviously depends on the frequency. We argue that the lowest frequencies which are accessible, or compatible with, the experiment are relevant for the determination of the gel point. The criterion that we propose is based on the detection of a non-zerorelaxed-elastic modulus which clearly indicates that the theoretical gel point (infinite viscosity and zero elasticity) has «just» been passed. In a kinetic process the precision is limited by the finite number of measurements. So, one has to allow for a certain time interval in the location of the gel point. However,we have noticed in our experiments that this time interval may correspond only to minor differences, when evaluated in terms of fraction of reacted bonds : for instance, of the order of $0.1 \%$ as compared to $7 \%$ for the helix amount.

We summarize these observations in figure 9. The time dependence of $G^{\prime}$ and $G^{\prime \prime}$ in a kinetic gelation process is plotted for the lowest frequency which is compatible with the kinetics $(f \approx 0.01 \mathrm{~Hz})$. The crossing of $G^{\prime}$ and $G^{\prime \prime}$ determines practically the gelation time $t_{\mathrm{g}}$. Besides, an independent investigation is necessary to follow the transformation, at a microscopic level. So, one is able to associate to the gel time $t_{\mathrm{g}}$, a critical degree of conversion $\chi_{\mathrm{c}}$ (helix content for gelatin). On both sides of this threshold, gelling systems show long relaxation times which prevent from measuring the zero-frequency limit of 
viscosity and elasticity, for a certain range of the transformation (for instance, in our case, for $\left.|\Delta \chi| / \chi_{\mathrm{c}}<0.1\right)$.

Again, in a kinetic experiment, repeated mechanical deformations should be avoided during this period, as they might lead to several drawbacks, such as :

(i) break of some junctions in a physical gel,

(ii) build in residual stresses, which do not have time to relax, before the system has evolved.

In this viscoelastic range, below the gel point, the static viscosity is expected to be higher than the ratio $G^{\prime \prime} / 2 \pi f$, while above the gel point, the relaxed elastic modulus is to be lower than $G^{\prime}$, as it appears from the spectra $G^{\prime \prime}(f)$ and $G^{\prime}(f)$.

When one excludes this range, we have shown that for :

$$
0.1 \leqslant|\Delta \chi| / \chi_{\mathrm{c}} \leqslant 0.3 \text { to } 0.5
$$

we are able to explore the relaxed regimes. So, we indicate in figure 9 the Newtonian regime, in the sol phase, and the relaxed regime for the shear modulus, in the gel phase. In this range, one may search the validity of the percolation theory. The width of the critical region depends on the nature of the process, and can be estimated theoretically by applying the Ginzburg argument. For instance, in a vulcanization process in semi-dilute solutions, Daoud [22] has shown that the critical region $\frac{|\Delta p|^{*}}{p_{c}}$ is a function of the ratio $c / c^{*}$ of the polymer concentration $c$ to the overlap concentration $c^{*}$ :

$$
\frac{|\Delta p|^{*}}{p_{\mathrm{c}}} \sim\left(\frac{c}{c^{*}}\right)^{-5 / 12}
$$

The critical region, where the percolation theory applies, is broader close to the overlap concentration $c^{*}$. Out of these limits, the classical, mean field exponents should be observed.

If we may try a comparison with our gel, we should consider $c^{*} \approx 0.5 \% \mathrm{~g} / \mathrm{g}$, which for $c \approx 5 \% \mathrm{~g} / \mathrm{g}$ (our measurements) gives a critical region of the order of 0.38 .

Universal behaviours are expected for the static viscosity $\eta$ and the relaxed elasticity $E$ in the critical region. The exact amplitudes of $\eta$ and $E$ depend on the local properties of each system. By extrapolating the log-log plots of $G^{\prime}$ and $G^{\prime \prime}$, towards the close vicinity of the gel point $(|\Delta \chi| /<0.1)$, one may derive the divergence of viscosity, or the increase of elasticity, which are represented with dashed lines in figure 9. By studying non kinetical processes of gelation, one should be able to reduce this « unknown » range, but it will not be completely suppressed. The same stands for the determination of the gel point itself.

Our investigation of the gelation process of gelatin gels establishes a firm analogy between the physical gelation and the chemical gelation. Despite the complexity of the local structure of physical gels, the percolation theory provides a clear framework for analysing the rheological modifications, in the course of gelation. The physical junctions appear as « permanent » crosslinks formed at random.

We have shown that, within our experimental conditions the kinetics of gelation has no influence upon the relation between the rheological properties and the structural parameter $\chi$. However, in the critical region, time and $\chi$ parameter are not simply proportional. Kinetic experiments on gelation of silica particles have been recently reported by Gauthier-Manuel et al. [23]. The rheological parameters were shown to be critically time dependent. This was supposed to be equivalent to the crosslinking critical dependence. We establish that this equivalence cannot be generalized without further justification.

The percolation theory has also ben used to interpret the rheological measurements in a gelation process analogous to vulcanization (hydrolyzed polyacrylamide chains crosslinked by Chromium (III) ions in solution). In this system both kinetic and static measurements are reported, which are in agreement with the percolation theory [24].

To generalyze our results on gelatin gels, it should be necessary to extend the procedure to different concentrations and a wider range of temperatures. The experimental limitations have to be considered. Low temperatures give more rapid, but less reproducible gelations $\left(t_{g}<30 \mathrm{~min}\right)$. For different concentrations, close to $c^{*}(c \approx 1 \% \mathrm{~g} / \mathrm{g})$, the critical region should be broader. But the gels are softer, and may show structural defects.

This paper indicates a procedure for analysing a kinetic gelation process. The independent measurement of the conversion factor allows an analysis which eliminates explicitly the time dependence of the phenomenon. When the experiment is repeated in different conditions, one is able to show whether the kinetics has an influence on the critical behaviour, for a given system. This should help to elucidate the role of the kinetic assumptions in the various theoretical models which have been proposed so far. 


\section{References}

[1] De Gennes, P. G., Scaling concepts in polymer physics (Cornell Univ. Press) 1979.

[2] Stauffer, D., Phys. Rep. 54 (1974) 1.

[3] Schosseler, F. and Leibler, L., Macromolecules 18 (1985) 398.

[4] Bouchaud, E., Delsanti, M., Adam, M., Daoud, M., Durand, D., J. Phys. France 47 (1986) 1273.

[5] Kantor, Y. and Webman, I., Phys. Rev. Lett. 52 (1984) 1891.

[6] Gauthier-Manuel, B., Thèse d'état, Paris (1986).

[7] Chambon, F. and Winter, H. H., Polym. Bull. 13 (1985) 499.

[8] Adam, M., Delsanti, M., Durand, D., Macromolecules 18 (1985) 2285.

[9] Tокiта, M., Niki, R., Hikichi, K., J. Chem. Phys. 83 (1985) 2583.

[10] Djabourov, M., Maquet, J., Théveneau, H., Leblond, J., PAPON, P., Br. Polym. J. 17 (1985) 169.

[11] See for instance WARD, I. M., Mechanical properties of solid polymers (J. Wiley Ed.) 1979.

[12] See for instance ZALLEN, R., The physics of amorphous solids (J. Wiley and sons, N.Y.) 1983.
[13] Stauffer, D., Coniglio, A., Adam, M., $A d v$. Polym. Sci. 44 (1982) 103.

[14] De Gennes, P. G., C. R. Hebd. Séan. Acad. Sci. 286B (1980) 131.

[15] De Gennes, P. G., J. Phys. Lett. 37 (1976) L1.

[16] FloRY, P. J., The principles of polymer chemistry (Cornell Univ. Press) 1953.

[17] Gordon, M., Ross-Murphy, S. B., J. Phys. A 12 (1979) L155.

[18] Derrida, B., Stauffer, D., Herrmann, H. J., Vannimenus, J., J. Phys. Lett. 44 (1983) L701.

[19] Herrmann, H. J., Stauffer, D., Landau, D. P., J. Phys. A 16 (1983) 1221.

[20] Mattews-Morgan, D., Landau, D. P., HerrMANN, H. J., Phys. Rev. B 29 (1984) 6328.

[21] Ziff, R. M., Hendricks, E. M., ERnSt, M. H., Phys. Rev. Lett. 49 (1982) 593.

[22] Daoud, M., J. Phys. Lett. 40 (1979) L201.

[23] Gauthier-Manuel, B., Guyon, E., Roux, S., Gits, S., Lefaucheux, F., J. Phys. France 48 (1987) 869.

[24] Salomé, L., Thèse, Paris (1987). 